\title{
THE DEGREE OF APPROXIMATION BY CHEBYSHEVIAN SPLINES
}

BY

\author{
R. DEVORE( (1) AND F. RICHARDS
}

ABSTRACT. This paper studies the connections between the smoothness of a function and its degree of approximation by Chebyshevian splines. This is accomplished by proving companion direct and inverse theorems which give a characterization of smoothness in terms of degree of approximation. A determination of the saturation properties is included.

1. Introduction. The purpose of this paper is to study the relation between the smoothness of a function and its degree of approximation by Chebyshevian splines. Many such results are known, especially, for algebraic polynomial splines with equally spaced knots. The most common type of result is one which gives an estimate for the degree of approximation by a method of spline approximation in terms of the smoothness of the function. This type of estimate is customarily called a direct theorem of approximation. Our main interest lies in the opposite direction. Namely, when a certain rate of approximation is known, what can be said about the smoothness of the function being approximated? We settle this this problem for Chebyshevian splines, with the knots satisfying a certain mixing condition.

Let $u_{0}, \cdots, u_{k-1}$ be $k$ times continuously differentiable functions on $[0,1]$ $\left(u_{i} \in C^{(k)}[0,1]\right)$ which form an extended complete Chebyshev (E.C.T.) system on $[0,1]$. We refer the reader to either of the books S. Karlin and W. J. Studd en [5] or S. Karlin [4] for the definition and fundamental properties of E.C.T. systems. We assume that the $u_{i}$ are the canonical basis represented by

$$
\begin{aligned}
& u_{0}(x)=w_{0}(x) \\
& u_{1}(x)=w_{0}(x) \int_{0}^{x} w_{1}\left(\xi_{1}\right) d \xi_{1}
\end{aligned}
$$

$$
u_{k-1}(x)=w_{0}(x) \int_{0}^{x} w_{1}\left(\xi_{1}\right) \int_{0}^{\xi_{1}} w_{2}\left(\xi_{2}\right) \ldots \int_{0}^{\xi_{k-2}} w_{k-1}\left(\xi_{k-1}\right) d \xi_{k-1} \ldots d \xi_{1},
$$

Received by the editors May 31, 1972 and, in revised form, September 10, 1972. AMS (MOS) subject classifications (1970). Primary 41A15, 41A25, 41A40.

Key words and phrases. Splines, degree of approximation, Chebyshev system, saturation, inverse the orems.

(1) The first author gratefully acknowledges NSF support in Grant GP 19620. 
where the functions $w_{i}$ are strictly positive on $[0,1]$ and $w_{i} \in C^{(k-i)}[0,1]$. If we let $U_{k-1}$ denote the span of $\left\{u_{0}, \cdots, u_{k-1}\right\}$, then $U_{k-1}$ is the null space of the differential operator

$$
L=D_{k-1} \cdots D_{0} \quad \text { where } D_{i}(f)=\left(f / w_{i}\right)^{\prime} .
$$

A function $S$ is said to be a Chebyshevian spline if there are points $0=x_{0}$ $<x_{1}<\cdots<x_{m-1}<x_{m}=1$, such that on each interval $\left[x_{i-1}, x_{i}\right), i=1, \cdots, m$, $S$ is in $U_{k-1}$. The points $x_{i}$ are called the knots of the spline. We make no restriction on the continuity of $S$ at the knots.

If $\delta=\left\{0=x_{0}<x_{1}<\cdots<x_{m-1}<x_{m}=1\right\}$, let $\delta(\delta)$ denote the collection of Chebyshevian splines whose knots are contained in $\delta$. Define the error in approximating $f$ by $S(\delta)$ as

$$
E_{\delta}(f)=\inf _{S \in \mathcal{S}(\varepsilon)}\|f-S\|
$$

where $\|\cdot\|$ denotes he supremum norm on $[0,1]$.

Now, suppose $\left(\delta_{n}\right)$ is a sequence of sets of knots $\delta_{n}=\left\{0=x_{0}^{(n)}<x_{1}^{(n)}<\right.$ $\left.\cdots<x_{m_{n}-1}^{(n)}<x_{m_{n}}^{(n)}=1\right\}$. We let

$$
\left\|\delta_{n}\right\|=\max _{1 \leq i \leq m_{n}}\left|x_{i}^{(n)}-x_{i-1}^{(n)}\right| .
$$

In order to guarantee that $E_{\delta_{n}}(f) \rightarrow 0$ for each $f \in C[0,1]$, we assume that $\left\|\delta_{n}\right\| \rightarrow 0 \quad(n \rightarrow \infty)$.

For an integer $r \geq 0$, let $\Delta_{t}^{r}$ denote the $r$ th order difference operator with step size $t$,

$$
\Delta_{t}^{r}(f, x)=(-1)^{r} \sum_{j=0}^{r}(-1)^{j}\left(\begin{array}{l}
r \\
j
\end{array}\right) f(x+j t)
$$

The corresponding $r$ th order modulus of smoothness of $f$ is given by

$$
\omega_{r}(f, b)=\sup _{0 \leq t \leq b}\left\|\Delta_{t}^{r}(f, x)\right\|[0,1-r t] .
$$

The notation $\|\cdot\|[a, b]$ is used to indicate that the norm is taken over $[a, b]$, and is thus the supremum on $[a, b]$. When $[a, b]$ is omitted, the norm is understood to be on $[0,1]$.

It is relatively easy to establish the estimate that if $0<a \leq k$ and $\omega_{k}(f, b)$ $=O\left(b^{a}\right)(b \rightarrow 0)$

$$
E_{\delta_{n}}(f)=O\left(\left\|\delta_{n}\right\|^{a}\right) \quad(n \rightarrow \infty)
$$


We do this in $\$ 4$, where in fact we show that the estimate in (1.7) can actually be obtained by using splines $S_{n} \in S\left(\delta_{n}\right)$ with $S_{n} \in C^{(k-2)}[0,1]$. Our method of proof is a straightforward extension of the techniques given by V. Popov and B. Sendov [8], who gave similar estimates for algebraic polynomial splines.

What is of primary interest to us is in what sense are the estimates of (1.7) the best possible? More precisely we ask the following two questions: When does (1.7) imply that $\omega_{k}(f, b)=O\left(b^{\alpha}\right)$; i.e., does the inverse theorem to (1.7) hold? Secondly, is it possible to improve (1.7) if we assume higher smoothness for $f$ ?

It is not possible to answer these questions without some additional restrictions on the sets of knots. The simplest way to see this is when a fixed point, say $1 / 2$, appears in each set $\delta_{n}$. Then, any Chebyshevian spline $S$ which has a single knot at $1 / 2$ will have $E_{\delta_{n}}(S)=0, n=1,2, \cdots$, while of course $S$ need not even be continuous. More generally, the same phenomenon manifests itself when a fixed point falls only in small intervals (in comparison to $\left.\left\|\delta_{n}\right\|\right)$. In order to avoid this, we will require that the sequence of sets $\left(\delta_{n}\right)$ satisfies the following mixing condition.

(1.8) There is a constant $\rho>0$, with the property that whenever $n>0$ and $1 \leq i \leq m_{n}-1$, then there is an $n^{\prime}>n$ such that $x_{j}^{\left(n^{\prime}\right)}<x_{i}^{(n)}<x_{j+1}^{\left(n^{\prime}\right)}$ with

$$
\min \left(\left|x_{j}^{\left(n^{\prime}\right)}-x_{i}^{(n)}\right|,\left|x_{j+1}^{\left(n^{\prime}\right)}-x_{i}^{(n)}\right|\right)>\rho\left\|\delta_{n}\right\| .
$$

It is easy to see that $(1.8)$ guarantees that the following holds.

(1.9) There is a constant $\rho>0$, with the property that whenever $n>0$ and $x \in\left[0,1-\rho\left\|\delta_{n}\right\|\right]$, there is an $n^{\prime} \geq n$ such that $x_{j}^{\left(n^{\prime}\right)} \leq x<x_{j+1}^{\left(n^{\prime}\right)}$ with $\left|x-x_{j+1}^{\left(n^{\prime}\right)}\right|$ $\geq \rho\left\|\delta_{n}\right\|$.

It is important to point out that the mixing condition puts a restriction on how fast $\left(\left\|\delta_{n}\right\|\right)$ can tend to 0 , since, for each $n$, there must exist an $n^{\prime}>n$ with $\left\|\delta_{n^{\prime}}\right\| \geq \rho\left\|\delta_{n}\right\|$. We can put this in the following form for later use.

Under condition (1.8), we have for each $n$

$$
\sup _{\nu \geq n+1}\left\|\delta_{\nu}\right\| \geq \rho\left\|\delta_{n}\right\|
$$

With the added restriction (1.8), we are able to establish in $\$ 3$, that if $E_{\delta_{n}}(f)=O\left(\left\|\delta_{n}\right\|^{\alpha}\right)(n \rightarrow \infty)$, then $\omega_{k}(f, b)=O\left(b^{a}\right)(b \rightarrow 0)$. This answers our first question. With regard to the second question, we show in $\$ 3$ that if $E_{\delta_{n}}(f)$ $=O\left(\left\|\delta_{n}\right\|^{k}\right)$, then $f \in U_{k-1}$. This is the saturation phenomenon, i.e., the estimates of (1.7) cannot be improved by assuming higher smoothness for $f$. Our results show that Chebyshevian splines are saturated with order $\left(\left\|\delta_{n}\right\|^{k}\right)$ and saturation class $\left\{f: \omega_{k}(f, b)=O\left(b^{k}\right)\right\}$ (see G. Lorentz [6] for a discussion of the 
concept of saturation). Since our inverse and saturation theorems are proved with no continuity restrictions at the knots, they apply to any spline approximation method provided the mixing condition on the knots holds.

The most important case covered by our results is for algebraic polynomial splines; $u_{i}(x)=x^{i}, i=0, \cdots, k-1$. The saturation and inverse theorems for algebraic polynomial splines with equally spaced knots (i.e., $\left.\delta_{n}=(i / n)_{i=0}^{n}\right)$ were first shown by K. Scherer [10]. Saturation theorems, in this case, were given independently by D. Gaier [3] (for "o") and F. Richards [9] (see also G. Butler and F. Richards [1] for saturation in $L_{p}$ spaces). We should also point out that Scherer's results apply only to smooth splines (i.e. $S \in C^{(k-2)}$ ) while in [3] and [9] no smoothness condition at the knots is needed.

The techniques developed here, besides having more general applications, are also simpler than those developed in [9], [10], when we restrict our attention to algebraic polynomial splines. In $\$ 5$, we give a finer description of the saturation class for approximation by algebraic polynomial splines with equally spaced knots by establishing an asymptotic formula of Voronovskaja type.

Our main tool is divided differences. To handle the general case of Chebyshevian splines, we need to develop some properties of generalized divided differences, which is done in $\$ 2$. The reader interested only in algebraic polynomial splines can skip $\$ 2$ and proceed directly to $\$ 3$, in which case the generalized divided difference $f(x, \cdots, x+k t)$ is to be interpreted as

$$
f(x, \cdots, x+k t)=t^{-k} \Delta_{t}^{k}(f, x) / k !
$$

2. Generalized divided differences. We will need to use the concept of generalized divided differences (see Karlin [4, p. 521]). Let $u_{k}$ be defined by

$$
u_{k}(x)=w_{0}(x) \int_{0}^{x} w_{1}\left(\xi_{1}\right) \int_{0}^{\xi_{1}} w_{2}\left(\xi_{2}\right) \ldots \int_{0}^{\xi_{k-1}} w_{k}\left(\xi_{k}\right) d \xi_{k} \ldots d \xi_{1}
$$

where $w_{k}(x)=1$ on $[0,1]$. Then $L u_{k}=1$ on $[0,1]$ and $\left\{u_{0}, \cdots, u_{k}\right\}$ is also an E.C.T. system.

If $0 \leq x_{0}<x_{1}<\cdots<x_{k} \leq 1$ and $f \in C[0,1]$, then the generalized divided difference of $f$ at $x_{0}, \ldots, x_{k}$ is defined by

$$
f\left(x_{0}, x_{1}, \cdots, x_{k}\right)=U\left(\begin{array}{cccc}
x_{0} & \cdots & x_{k-1} & x_{k} \\
u_{0} . & \cdots & u_{k-1} & f
\end{array}\right) / U\left(\begin{array}{lll}
x_{0} & \cdots & x_{k} \\
u_{0} & \cdots & u_{k}
\end{array}\right)
$$

where

$$
U\left(\begin{array}{lll}
x_{0} & \cdots & x_{k} \\
g_{0} & \cdots & g_{k}
\end{array}\right)=\operatorname{det}\left(g_{i}\left(x_{j}\right)\right)_{i, j=0}^{k}
$$


As long as the points $x_{i}$ are distinct, the denominator in (2.2) does not vanish. It is clear that if $f \in \operatorname{sp}\left(u_{0}, \cdots, u_{k-1}\right)$, then $f\left(x_{0}, \cdots, x_{k}\right)=0$ for any $0 \leq x_{0}$ $<\cdots<x_{k} \leq 1$.

Let $\phi_{k-1}$ denote the fundamental spline

$$
\begin{aligned}
\phi_{k-1}(x ; t) & =0, \quad x<t, \\
= & w_{0}(x) \int_{t}^{x} w_{1}\left(\xi_{1}\right) \int_{t}^{\xi_{1}} w_{2}\left(\xi_{2}\right) \\
& \quad \ldots \int_{t}^{\xi_{k-2}} w_{k-1}\left(\xi_{k-1}\right) d \xi_{k-1} \cdots d \xi_{1}, \quad x \geq t .
\end{aligned}
$$

The $B$-spline $M\left(t ; x_{0}, \cdots, x_{k}\right)$ for the knots $0 \leq x_{0}<x_{1}<\cdots<x_{k} \leq 1$ is defined as the generalized divided difference of $\phi_{k-1}(x ; t)$ with respect to the first variable. Notationally,

$$
M\left(t ; x_{0}, \ldots, x_{k}\right)=\phi_{k-1}\left(x_{0}, \ldots, x_{k} ; t\right) .
$$

The $B$-spline $M\left(t ; x_{0}, \cdots, x_{k}\right)$ has the following fundamental properties:

$$
\begin{aligned}
& M\left(t ; x_{0}, \ldots, x_{k}\right) \text { is nonnegative on }[0,1] \\
& \text { and vanishes outside }\left[x_{0}, x_{k}\right] . \\
& \int_{0}^{1} M\left(\xi ; x_{0}, \ldots, x_{k}\right) d \xi=1 .
\end{aligned}
$$

As in the case of ordinary divided differences, we have the Peano formula

$$
f\left(x_{0}, \ldots, x_{k}\right)=\int_{0}^{1} L f(\xi) M\left(\xi ; x_{0}, \ldots, x_{k}\right) d \xi
$$

whenever $f^{(k-1)}$ is absolutely continuous and $L f \in L_{1}[0,1]$. On this point, there is a misprint on p. 523 of Karlin [4], where it is stated that (2.8) holds but with the adjoint operator $L^{*}$ in place of $L$. The identity $(2.8)$ is easily established for functions $f$ with $f^{(k-1)}$ absolutely continuous and $L f$ continuous. The general case is handled by taking a sequence of functions $\left(f_{n}\right)$ in $C^{(k)}[0,1]$ which converge uniformly to $f$ and $L f_{n} \rightarrow L f$ in $L_{1}[0,1]$.

When $f \in C[0,1]$, we define the generalized modulus of continuity $\omega^{*}(f, b)$ by

$$
\omega^{*}(f, b)=\sup _{0 \leq t \leq b} t^{k}\|f(x, x+t, \cdots, x+k t)\|[0,1-k t] .
$$

Our main objective in this section is to show how $\omega^{*}(f, b)$ can be characterized in terms of ordinary smoothness as described by $\omega_{k}(f, b)$. We first establish an identity between generalized divided differences and ordinary divided differences. 
Let $A(x, t)$ denote the determinant in the denominator of $(2.2)$ for $x_{i}=x+i t$, $i=0, \cdots, k$. For the numerator in the expression (2.2) for $f(x, \cdots, x+k t)$, we add $(-1)^{j(k)}{ }_{j}$ times the $j$ th column to the 0 th column for $j=1,2, \cdots, k$. Note, we have indexed the columns and rows from 0 to $k$. In this way, the determinant remains the same but the 0 th column now has $(-1)^{k} \Delta_{t}^{k}\left(u_{i}, x\right)$ as its entry in the $i$ th row, $0 \leq i \leq k-1$, and $(-1)^{k} \Delta_{t}^{k}(f, x)$ for its entry in the $k$ th row. In a similar way, we can modify the suceeding columns to find

$$
U\left(\begin{array}{cccc}
x & \cdots & x+(k-1) t & x+k t \\
& & & f
\end{array}\right)=\operatorname{det}\left((-1)^{k-j} \Delta_{t}^{k-j}\left(g_{i}, x+j t\right)\right)_{i, j=0}^{k}
$$

where $g_{i}=u_{i}, i=0, \cdots, k-1$ and $g_{k}=f$. Note that $\Delta_{t}^{0}(g, x)$ is understood to be $g(x)$.

Now, we expand the right-hand side of (2.10) about the last row to find

$$
f(x, x+t, \cdots, x+k t)=(A(x ; t))^{-1} \sum_{r=0}^{k} \Delta_{t}^{k-r}(f, x+r t) A_{r}(x ; t)
$$

with

$$
A_{r}(x ; t)=\operatorname{det}\left((-1)^{k-j} \Delta_{t}^{k-j}\left(u_{i}, x+j t\right)\right){ }_{i=0 ; j=0, j \neq r}^{k-1, k} .
$$

The determinant $A(x ; t)$ can be written in a similar way to (2.12), namely

$$
A(x ; t)=\operatorname{det}\left((-1)^{k-j} \Delta_{t}^{k-j}\left(u_{i}, x+j t\right)\right)_{i, j=0}^{k} .
$$

Our first lemma determines the behavior of $A(x ; t)$ and $A_{r}(x ; t)$ as $t \rightarrow 0$. Denote by $W\left(\phi_{1}, \cdots, \phi_{r}\right)$ the Wronskian of $\phi_{1}, \cdots, \phi_{r}$.

Lemma 1. Let $\mu=1+2+\cdots+k$. Then

$$
(-1)^{\mu} \lim _{t \rightarrow 0} t^{-\mu} A(x ; t)=W\left(u_{0}, \cdots, u_{k}\right)(x) \geq B>0
$$

uniformly in $0 \leq x<1$. Also for each $r=0,1, \cdots, k$

$$
\lim _{t \rightarrow 0} t^{-\mu+k-r} A_{r}(x ; t)=(-1)^{\mu+k-r} \operatorname{det}\left(u_{i}^{(k-j)}(x)\right)_{i=0, j=0, j \neq r}^{k-1, k}
$$

uniformly in $0 \leq x<1$.

Remark. In particular, $(-1)^{\mu+k} \lim _{t \rightarrow 0} t^{-\mu+k} A_{0}(x ; t)=W\left(u_{0}, \ldots, u_{k-1}\right)(x)$ $\geq B>0$ uniformly in $0 \leq x<1$.

Proof. Suppose $0 \leq r \leq k$ and consider $A_{r}(x ; t)$ which is given by (2.12). We have from (2.12) that 


$$
t^{-\mu+k-{ }^{\prime}} A_{r}(x ; t)=\operatorname{det}\left((-1)^{k-j} \Delta_{t}^{k-j}\left(u_{i}, x+j t\right) / t^{k-j}\right)_{i=0, j=0, j \neq r}^{k-1, k}
$$

where we have divided each column by $t^{k-j}$ to compensate for the factor $t^{-\mu+k-r}$. Since $u_{i} \in C^{k}[0,1]$, for each $i, j$ there is a point $\xi_{i, j} \in(x, x+k t)$ for which $\Delta_{t}^{k-j}\left(u_{i}, x+j t\right) / t^{k-j}=u_{i}^{(k-j)}\left(\xi_{i, j}\right)$. Therefore, as $t \rightarrow 0$, this quantity converges to $u_{\cdot i}^{(k-j)}(x)$ uni formly for $0 \leq x<1$. This shows (2.15), where we have factored out the terms $(-1)^{k-j}$.

In the same way, we see that

$$
(-1)^{\mu} \lim _{t \rightarrow 0} t^{-\mu} A(x ; t)=\operatorname{det}\left(u_{i}^{(k-j)}(x)\right)_{i, j=0}^{k}=W\left(u_{0}, \cdots, u_{k}\right)(x) .
$$

It is shown in Karlin [4, p. 278] that $W\left(u_{0}, \cdots, u_{k}\right)(x)=\left(w_{0}(x)\right)^{k+1}\left(w_{1}(x)\right)^{k} \ldots$ $\left(w_{k-1}(x)\right)^{2}$ and $W\left(u_{0}, \cdots, u_{k-1}\right)(x)=\left(w_{0}(x)\right)^{k}\left(w_{1}(x)\right)^{k-1} \cdots\left(w_{k-1}(x)\right)$. Clearly both these quantities are positive on $[0,1]$.

Lemma 2. There is a constant $C>0$ such that for each $f \in C[0,1], t>0$, $x_{0} \in[0,1-k t]$, we bave $t^{k}\left|f\left(x_{0}, \cdots, x_{0}+k t\right)\right| \leq C\|f\|\left[x_{0}, x_{0}+k t\right]$.

Proof. It follows from Lemma 1 that there is a constant $C_{1}>0$ such that, for each $r=0,1, \ldots, k,\left\|t^{k-r}(A(x ; t))^{-1} A_{r}(x ; t)\right\|[0,1] \leq C_{1}, 0 \leq t \leq 1$, where the norm is taken with respect to the variable $x$ and the constant $C_{1}$ can be chosen independent of $r$ and $t$. Using this in (2.11), we find

$$
t^{k}\left|f\left(x_{0}, x_{0}+t, \cdots, x_{0}+k t\right)\right| \leq C_{1} \sum_{r=0}^{k} t^{r}\left|\Delta_{t}^{k-r}\left(f, x_{0}+r t\right)\right| \leq C_{2}\|f\|\left[x_{0}, x_{0}+k t\right]
$$

for some constant $C_{2}>0$. Here, we have used the fact that $\left|\Delta_{t}^{r}\left(f, x_{0}+r t\right)\right| \leq$ $2^{r}\|f\|\left[x_{0}, x_{0}+k t\right]$.

Lemma 3. If $0<\alpha \leq k$ and $f \in C[0,1]$, then

$$
\omega^{*}(f, t)=O\left(t^{a}\right) \quad(t \rightarrow 0)
$$

if and only if

$$
\omega_{k}(f, t)=O\left(t^{a}\right) \quad(t \rightarrow 0) .
$$

Proof. First suppose that (2.18) holds. Then, using (2.11) together with Lemma 1, we find that

$$
\begin{aligned}
\|f(x, x+t, \cdots, x+k t)\|[0,1-k t] & \leq \sum_{r=0}^{k} \omega_{k-r}(f, t)\left\|\frac{A_{r}(x ; t)}{A(x ; t)}\right\| \\
& \leq C_{3} t^{-k} \sum_{r=0}^{k} t^{r} \omega_{k-r}(f, t)
\end{aligned}
$$


with $C_{3}>0$ an absolute constant. From our assumption (2.18), it follows (see Timan $[11$, p. 107$])$ that as $t \rightarrow 0$.

$$
\omega_{\nu}(f, t)= \begin{cases}O\left(t^{a}\right), & \nu>a \\ O\left(t^{a} \ln t\right), & \nu=a \\ O\left(t^{\nu}\right), & \nu<a\end{cases}
$$

Using (2.20) in (2.19), we find for $0<a<k$ that

$$
\begin{aligned}
t^{k} \| f(x, x+ & +t, \ldots, x+k t) \|[0,1-k t] \\
& =O\left(\sum_{k-r>a} t^{a+r}+\sum_{k-r=a} t^{k} \ln t+\sum_{k-r<a} t^{k}\right)=O\left(t^{a}\right) \quad(t \rightarrow 0)
\end{aligned}
$$

as desired. When $\alpha=k$, then, because of (2.20),

$$
t^{k}\|f(x, x+t, \cdots, x+k t)\|[0,1-k t]=O\left(\omega_{k}(f, t)+\sum_{r=1}^{k} t^{k}\right)=O\left(t^{a}\right)
$$

as desired.

For the other direction, we again use (2.11) and find

$$
\Delta_{t}^{k}(f, x)=\frac{A(x ; t)}{A_{0}(x ; t)} f(x, \cdots, x+k t)-\sum_{r=1}^{k} \Delta_{t}^{k-r}(f, x+r t) \frac{A_{r}(x ; t)}{A_{0}(x ; t)} \cdot
$$

Therefore from Lemma 1

$$
\omega_{k}(f, t) \leq C_{4} t^{k}\|f(x, \cdots, x+k t)\|[0,1-k t]+\sum_{r=1}^{k} t^{r} \omega_{k-r}(f, t) .
$$

Now, suppose that $\omega^{*}(f, t)=O\left(t^{a}\right)$, so that $t^{k}\|f(x, \cdots, x+k t)\|[0,1-k t]=O\left(t^{a}\right)$. We want to show that $\omega_{k}(f, t)=O\left(t^{a}\right)$. This is clear when $\alpha \leq 1$, because the first term on the right hand side of $(2.21)$ is $O\left(t^{\alpha}\right)$ by assumption and the second sum is $O(t)$ because each term in the sum has a factor $t^{r}, r \geq 1$. Suppose we have established the result for all $\alpha \leq l, l$ an integer $1 \leq l \leq k-1$. If $l<a \leq l+1, a \neq k$ and $\omega^{*}(f, t)=O\left(t^{\alpha}\right)$, then by our induction hypothesis $\omega_{k}(f, t)=O\left(t^{l}\right)$. Therefore, using (2.20) in (2.21), we find

$$
\begin{aligned}
\omega_{k}(f, t) & =O\left(t^{a}+\sum_{k-r>l} t^{r+l}+\sum_{k-r=l}\left(t^{r+l} \ln t\right)+\sum_{k-r<l} t^{k}\right) \\
& =O\left(t^{a}+t^{l+1}+t^{k} \ln t+t^{k}\right)=O\left(t^{a}\right) \quad(t \rightarrow 0) .
\end{aligned}
$$

When $\alpha=k$, we have to use the additional information we have just obtained. For example, now we know $\omega_{k}(f, t)=O\left(t^{k-1 / 2}\right)$. Using this in (2.21), together with (2.20), we find $\omega_{k}(f, t)=O\left(t^{k}+\sum_{r=1}^{k} t^{k-r} \cdot t^{r}\right)=O\left(t^{k}\right)$.

3. Saturation and inverse theorems. Our main result is the following inverse theorem for approximation by Chebyshevian splines. 
Theorem 1. Let $\left(\delta_{n}\right)$ be a sequence of sets of knots with $\left\|\delta_{n}\right\| \rightarrow 0$ and $\left(\delta_{n}\right)$ satisfying the mixing condition (1.8). If $0<\alpha \leq k$ and $f \in C[0,1]$ with

$$
E_{\delta_{n}}(f) \leq M\left\|\delta_{n}\right\|^{a}, \quad n=1,2, \cdots,
$$

then $\omega_{k}(f, t)=O\left(t^{\alpha}\right)(t \rightarrow 0)$.

Proof. Because of Lemma 3, it is enough to show that

$$
\omega^{*}(f, t)=O\left(t^{\alpha}\right) \quad(t \rightarrow 0) .
$$

We will show first that (3.2) is valid for any $t$ of the form

$$
\begin{aligned}
& t=t_{\lambda_{n}}=k^{-1} \rho\left\|\delta_{\lambda_{n}}\right\|, \\
& \left\|\delta_{\lambda_{n}}\right\|=\sup _{\nu \geq \lambda_{n}}\left\|\delta_{\nu}\right\|,
\end{aligned}
$$

where (3.4) is used to define the sequence $\left(\lambda_{n}\right)$. Let $t$ be of the form (3.3) and $x_{0}$ be any point in $[0,1-k t]$. Then because of (1.9), we can find an $n^{\prime} \geq \lambda_{n}$ for which $x_{j}^{\left(n^{\prime}\right)} \leq x_{0}<x_{j+1}^{\left(n^{\prime}\right)}$ and $\left|x_{j+1}^{\left(n^{\prime}\right)}-x_{0}\right|>\rho\left\|\delta_{\lambda_{n}}\right\|$.

From our assumption (3.1), it follows that there is a spline $S_{n^{\prime}}$ in $S\left(\delta_{n^{\prime}}\right)$ for which $\left\|f-S_{n^{\prime}}\right\| \leq 2 M\left\|\delta_{n^{\prime}}\right\|^{a}$. Then because of (3.3), if $0<b \leq t$, the points $x_{0}, x_{0}+b, \ldots, x_{0}+k b$ all lie in the interval $\left[x_{j}^{\left(n^{\prime}\right)}, x_{j+1}^{\left.\left(n^{\prime}\right)\right)}\right.$ and, since $S_{n^{\prime}}$ is in $U_{k-1}$ on $\left[x_{j}^{\left(n^{\prime}\right)}, x_{j+1}^{\left(n^{\prime}\right)}\right), f\left(x_{0^{\prime}} x_{0}+b, \cdots, x_{0}+k b\right)=\left(f-S_{n^{\prime}}\right)\left(x_{0}, x_{0}+b, \cdots, x_{0}+k b\right)$.

From Lemma 2 and (3.3), we see that

$$
\omega^{*}(f, t)=\sup _{0 \leq b \leq t} b^{k}\left|f\left(x_{0}, x_{0}+b, \ldots, x_{0}+k b\right)\right| \leq C\left\|f-S_{n^{\prime}}\right\|\left[x_{0}, x_{0}+k b\right]
$$

$$
\leq 2 C M\left\|\delta_{n}\right\|^{a} \leq 2 C M\left\|\delta_{\lambda_{n}}\right\|^{a}=2 C M\left(k \rho^{-1} t\right)^{a}=C^{\prime} t^{a}
$$

with $C^{\prime}$ a constant independent of $t$. This establishes (3.2) for $t=t_{\lambda_{n}}$.

Now, it follows from our remark (1.10) that

$$
{ }^{t} \lambda_{n} / t_{\lambda_{n+1}}=\left\|\delta_{\lambda_{n}}\right\| /\left\|\delta_{\lambda_{n+1}}\right\| \leq \rho^{-1} .
$$

Hence, given any $t>0, t_{\lambda_{n+1}} \leq t<t_{\lambda_{n}}$ for some $n$ and $\omega^{*}(f, t) \leq \omega^{*}\left(f,{ }_{\lambda_{n}}\right) \leq$ $C^{\prime} t_{\lambda_{n}}^{\alpha} \leq C \rho^{-a_{\lambda^{\prime}}^{\alpha}} \leq C \rho^{-a_{t} a}$. This is (3.2) and the proof is complete.

In $\$ 4$, we will establish the direct theorem that $\omega_{k}(f, t)=O\left(t^{\alpha}\right)$ implies $E_{\delta_{n}}(f)=O\left(\left\|\delta_{n}\right\|^{a}\right)$. This gives the following corollary to Theorem 1.

Corollary 1. Let $\left(\delta_{n}\right)$ be a sequence of sets of knots satisfying the mixing condition (1.8). If $0<\alpha \leq k$ and $f \in C[0,1]$ then

$$
E_{\delta_{n}}(f)=O\left(\left\|\delta_{n}\right\|^{a}\right) \quad(n \rightarrow \infty)
$$

if and only if $\omega_{k}(f, t)=O\left(t^{\alpha}\right)(t \rightarrow 0)$. 
The following theorem is a " 0 " saturation theorem which shows that the estimate (3.7) cannot be improved by assuming higher smoothness for the function $f$.

Theorem 2. Let $\left(\delta_{n}\right)$ be a sequence of sets of knots satisfying the mixing condition (1.8). If $f \in C[0,1]$ and

$$
E_{\delta_{n}}(f)=o\left(\left\|\delta_{n}\right\|^{k}\right) \quad(n \rightarrow \infty)
$$

then $f \in U_{k-1}$.

Proof. From the representation (2.4), it follows that there is a constant $C>$ 0 such that

$$
\left|\phi_{k-1}(x ; \xi)\right| \leq C t^{k-1}, \quad|\xi-x| \leq k t_{0}
$$

Using this in Lemma 2, we find that

$$
|M(\xi ; x, \cdots, x+k t)|=\left|\phi_{k-1}(x, \ldots, x+k t ; \xi)\right| \leq C t^{-1}
$$

for all $x$ and $\xi$. Here, we have used the fact that $M$ vanishes for $\xi$ outside $[x, x+k t]$, and hence we needed only to estimate for $|\xi-x| \leq k t$ which is done by (3.9).

Now, suppose $f$ satisfies (3.8). From Theorem 1, we have that $\omega_{k}(f, t)=$ $O\left(t^{k}\right)$ and hence $f^{(k-1)}$ is absolutely continuous and $f^{(k)} \in L_{\infty}$. This gives that $L f \in L_{\infty}$ and therefore

$$
f(x, x+t, \ldots, x+k t)=\int_{x}^{x+k t} L f(\xi) M(\xi ; x, x+t, \ldots, x+k t) d \xi .
$$

Let $x_{0}$ be any Lebesgue point of $L f$, i.e., $b^{-1} \int_{x_{0}}^{x_{0}+b}\left|L f\left(x_{0}+t\right)-L f\left(x_{0}\right)\right| d t \rightarrow 0$ $(b \rightarrow 0)$. Thus, using (3.10), (2.6) and (2.7), we find

$$
\begin{aligned}
& \left|f\left(x_{0}, \ldots, x_{0}+k t\right)-L f\left(x_{0}\right)\right| \\
& \quad=\left|\int_{x_{0}}^{x_{0}+k t}\left(L f(\xi)-L f\left(x_{0}\right)\right) M\left(\xi ; x_{0}, x_{0}+t, \ldots, x_{0}+k t\right) d \xi\right| \\
& \quad \leq C t^{-1} \int_{x_{0}}^{x_{0}+k t}\left|L f(\xi)-L f\left(x_{0}\right)\right| d \xi \rightarrow 0 \quad(t \rightarrow 0) .
\end{aligned}
$$

Now, we can argue the same as in Theorem 1 to show that (3.8) implies that $\|f(x, \cdots, x+k t)\|[0,1-k t] \rightarrow 0(t \rightarrow 0)$. Using this with (3.11) shows that $L f\left(x_{0}\right)=0$ at each Lebesgue point $x_{0}$ and hence $L f=0$, a.e. Since $f^{(k-1)}$ is absolutely continuous, $f \in U_{k-1}$.

4. A direct theorem. In this section, we will establish a direct theorem which is a companion to Theorem 1 . However, for the direct results we do not 
need the mixing condition (1.8). Also, we will be able to establish our upper estimates with splines of continuity class $C^{(k-2)}[0,1]$.

We introduce the auxiliary E.C.T. systems for $\nu=0, \cdots, k-1$ :

(4.1)

$$
\begin{aligned}
& u_{0, \nu}(x)=w_{\nu}(x) \\
& u_{1, \nu}(x)=w_{\nu}(x) \int_{0}^{x} w_{\nu+1}\left(\xi_{1}\right) d \xi_{1}
\end{aligned}
$$

$$
\begin{aligned}
& u_{k-1-\nu, \nu}(x)=w_{\nu}(x) \int_{0}^{x} w_{\nu+1}\left(\xi_{1}\right) \int_{0}^{\xi} w_{\nu+2}\left(\xi_{2}\right) \\
& \cdots \int_{0}^{\xi_{k-\nu-2} w_{k-1}}\left(\xi_{k-\nu-1}\right) d \xi_{k-\nu-1} \cdots d \xi_{1} \text {. }
\end{aligned}
$$

Note that for $\nu=0$, we get our usual system. Also,

$$
u_{j+1, \nu-1}(x)=w_{\nu-1}(x) \int_{0}^{x} u_{j, \nu}(t) d t
$$

We define the new space of spline functions $S\left(\nu, \delta_{n}\right)$ to be the collection of all functions $S$ for which

$$
\begin{aligned}
& \text { on each interval }\left[x_{i-1}^{(n)}, x_{i}^{(n)}\right), i=1, \ldots, m_{n}, \\
& S \text { is in } \operatorname{sp}\left(u_{0, \nu}, u_{1, \nu}, \cdots, u_{k-1-\nu, \nu}\right)
\end{aligned}
$$

and

$$
S \in C^{(k-\nu-2)}[0,1]
$$

In particular, for $\nu=k-1$, there is no continuity assumption on the splines.

We begin with the following reduction lemma.

Lemma 4. Suppose $\left(\delta_{n}\right)$ is a sequence of sets of knots and $1 \leq \nu \leq k-1$. Given $f \in C[0,1]$, suppose $\epsilon_{n}>0$ and $S_{n, \nu} \in S\left(\nu, \delta_{n}\right)$ satisfy

$$
\left\|f-s_{n, \nu}\right\| \leq \epsilon_{n}, \quad n=1,2, \cdots
$$

Then, for any $F$ satisfying $D_{\nu-1}(F)=f$, there exists $S_{n, \nu-1} \in S\left(\nu-1, \delta_{n}\right)$ sat. isfying

$$
\left\|F-S_{n, \nu-1} ! \leq(2 k+1) \epsilon_{n}\right\| w_{\nu-1}\|\| \delta_{n} \|
$$

Proof. Let $M_{i, \nu}(t)$ be the $B$-splines with respect to $\left\{u_{0, \nu}, \cdots, u_{k-\nu-1, \nu}\right\}$, that is $M_{i, \nu}(t)=\phi_{k-\nu-1, \nu}\left(x_{i}^{(n)}, \ldots, x_{i+k-\nu}^{(n)} ; t\right), 0 \leq i \leq m_{n}-k+\nu$, where 


$$
\begin{aligned}
\dot{\phi}_{k-\nu-1, \nu}(x ; t) & =0, \quad x<t, \\
= & w_{\nu}(x) \int_{t}^{x} w_{\nu+1}\left(\xi_{1}\right) \int_{t}^{\xi_{1}} w_{\nu+2}\left(\xi_{2}\right) \\
& \quad \ldots \int_{t}^{\xi}{ }_{k-\nu-2} w_{k-1}\left(\xi_{k-\nu-1}\right) \quad d \xi_{k-\nu-1} \cdots d \xi_{1}, \quad x \geq t .
\end{aligned}
$$

As usual, $M_{i, \nu}$ is nonnegative on $[0,1]$, vanishes outside of $\left[x_{i}^{(n)}, x_{i+k-\nu}^{(n)}\right]$ and $\int_{0}^{1} M_{i, \nu}(t) d t \stackrel{i, \nu}{=} 1$.

Now, let

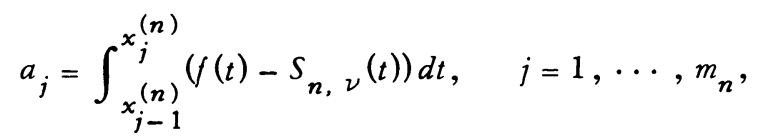

and set

$$
S_{n, \nu-1}(x)=w_{\nu-1}(x) \int_{0}^{x} S_{n, \nu}(t) d t+w_{\nu-1}(x) \sum_{j=0}^{m_{n}-k+\nu} a_{j} \int_{0}^{x} M_{i}(t) d t
$$

From (4.2), one easily verifies that $S_{n, \nu-1} \in \mathcal{S}\left(\nu-1, \delta_{n}\right)$.

First suppose $F_{1}(x)$ has the form $F_{1}(x)=w_{\nu-1}(x) \int_{0}^{x} f(t) d t$. If $x \in[0,1]$, say $x_{i}^{(n)}<x \leq x_{i+1}^{(n)}$, then

$$
\begin{aligned}
& F_{1}(x)-S_{n, \nu-1}(x)=\left.w_{\nu-1}(x) \int_{0}^{x} f(t)-S_{n, \nu}(t)\right) d t \\
&-w_{\nu-1}(x) \sum_{j=0}^{m} a_{j} \int_{0}^{x} M_{j, \nu}(t) d t \\
&=w_{\nu-1}(x)\left[\sum_{j=0}^{i} a_{j}+\int_{x_{i}^{(n)}}^{x}\left(f(t)-S_{n, \nu}(t)\right) d t\right.\left.-\sum_{j=0}^{i-k+\nu} a_{j}-\sum_{j=i-k+\nu+1}^{i} a_{j} \int_{0}^{x} M_{j, \nu}(t) d t\right] \\
&=w_{\nu-1}(x)\left[\sum_{j=i-k+\nu+1}^{i} a_{j}+\int_{x_{i}^{(n)}}^{x}\left(f(t)-S_{n, \nu}(t)\right) d t\right. \\
&\left.-\sum_{j=i-k+\nu+1}^{i} a_{j} \int_{0}^{x} M_{j, \nu}(t) d t\right] .
\end{aligned}
$$

The notation $\Sigma^{\prime}$ is used to indicate that the upper limit in the sum is to be replaced by $m_{n}-k+\nu$ when $i>m_{n}-k+\nu$. In the second inequality, we have used the fact that $M_{j, \nu}$ is supported on $\left[x_{j}^{(n)}, x_{j+k-\nu}^{(n)}\right]$ and $\int_{0}^{1} M_{j, \nu}(t) d t=1$.

From the definition of the $a_{j}$ 's, we find 


$$
\begin{aligned}
\sum_{j=i-k+\nu+1}^{i}\left|a_{j}\right| & \leq \int_{x_{i-k+\nu}^{(n)}}^{x_{i}^{(n)}}\left|f(t)-S_{n, \nu}(t)\right| d t \\
& \leq\left|x_{i}^{(n)}-x_{i-k+\nu}^{(n)}\right|\left\|f-S_{n, \nu}\right\| \leq k\left\|\delta_{n}\right\| \epsilon_{n} .
\end{aligned}
$$

Similarly,

$$
\left|\sum_{j=i-k+\nu+1}^{i} a_{j} \int_{0}^{x} M_{j, \nu}(t) d t\right| \leq \sum_{j=i-k+\nu+1}^{i}\left|a_{j}\right| \leq k\left\|\delta_{n}\right\| \epsilon_{n} .
$$

Also,

$$
\left|\int_{x_{i}^{(n)}}^{x}\left(f(t)-S_{n, \nu}(t)\right) d t\right| \leq \int_{x_{i}^{(n)}}^{x_{i+1}^{(n)}}\left|\left(f(t)-S_{n, \nu}(t)\right)\right| d t \leq\left\|\delta_{n}\right\| \epsilon_{n} .
$$

Using these last three estimates back in (4.9) shows that $\left|F_{1}(x)-S_{n, \nu-1}(x)\right| \leq$ $(2 k+1) \epsilon_{n}\left\|\delta_{n}\right\|\left\|w_{\nu_{-}}\right\|$. Since $x$ was arbitrary

$$
\left\|F_{1}-S_{n, \nu-1}\right\| \leq(2 k+1) \epsilon_{n}\left\|w_{\nu-1}\right\|\left\|\delta_{n}\right\|
$$

Finally, if $F$ is any function such that $D_{\nu_{-1}}(F)=f$, then $F=F_{1}+C w_{\nu_{-1}}$, with $C$ a constant, and thus the spline $S_{n, \nu-1}+C w_{\nu-1}$ provides the desired estimate (4.6).

Lemma 5. Suppose $f \in C[0,1], D_{k-1}(f) \in L_{\infty}$. Then, there exists a sequence of splines $\left(S_{n, k-1}\right)$, with $S_{n, k-1} \in \mathcal{S}\left(k-1, \delta_{n}\right)$ such that $\left\|f-S_{n, k-1}\right\| \leq$ $\left\|w_{k-1}\right\|\left\|\delta_{n}\right\|\left\|D_{k-1}(f)\right\|_{\infty}$.

Proof. From our assumptions, we have $f(x)=C w_{k-1}(x)+w_{k-1}(x) \int_{0}^{x} D_{k-1}(f)(t) d t$ with $C$ a constant.

Define $S_{n, k-1}$ by

$$
\begin{aligned}
S_{n, k-1}(x)=C w_{k-1}(x)+w_{k-1}(x) \int_{0}^{x} D_{k-1}(f)(t) d t, & \\
& x \in\left[x_{i}, x_{i+1}\right), i=0, \cdots, m_{n}-1 .
\end{aligned}
$$

Then, for $x \in\left[x_{i}, x_{i+1}\right)$

$$
\left|f(x)-S_{n, k-1}(x)\right|=\left|w_{k-1}(x) \int_{x_{i}}^{x} D_{k-1}(f)(t) d t\right| \leq\left\|w_{k-1}\right\|\left\|\delta_{n}\right\|\left\|D_{k-1}(f)\right\|_{\infty} .
$$

Since $x$ is arbitrary, $\left\|f-S_{n, k-1}\right\| \leq\left\|w_{k-1}\right\|\left\|\delta_{n}\right\|\left\|D_{k-1}(f)\right\|_{\infty}$ as desired.

Theorem 3. Let $\left(\delta_{n}\right)$ be a sequence of sets of knots with $\left\|\delta_{n}\right\| \rightarrow 0$. If $0<$ $\alpha \leq k$ and $f \in C[0,1]$ with $\omega_{k}(f, t)=O\left(t^{a}\right), t \rightarrow 0$, then there exists a sequence of splines $\left(S_{n}\right)$ with $S_{n} \in \mathcal{S}\left(0, \delta_{n}\right) \subseteq \mathcal{S}\left(\delta_{n}\right) \cap C^{k-2}[0,1]$ such that 


$$
\left\|f-S_{n}\right\|=O\left(\left\|\delta_{n}\right\|^{a}\right) \quad(n \rightarrow \infty) .
$$

In particular,

$$
E_{\delta_{n}}(f)=O\left(\left\|\delta_{n}\right\|^{a}\right) \quad(n \rightarrow \infty)
$$

Proof. First suppose that $\omega_{k}(f, t)=O\left(t^{k}\right)$ so that $f^{(k-1)}$ is absolutely continuous and $L f \in L_{\infty}$. Let $F=D_{k-2}, \cdots, D_{0}(f)$. Then, $D_{k-1}(F)=L f \in L_{\infty}$ and therefore from Lemma 5 , there exist splines $S_{n, k-1} \in \mathcal{S}\left(k-1, \delta_{n}\right), n=1,2, \cdots$, with $\left\|F-S_{n, k-1}\right\| \leq\left\|w_{k-1}\right\|\left\|\delta_{n}\right\|\|L(f)\|_{\infty}$. Now, we use the reduction lemma repeatedly to find that there are splines $S_{n, 0} \in \mathcal{S}\left(0, \delta_{n}\right), n=1,2, \cdots$, with

$$
\begin{aligned}
\left\|f-S_{n, 0}\right\| & \leq\left\|w_{0}\right\| \cdots\left\|w_{k-1}\right\|(2 k+1)^{k-1}\|L f\|_{\infty}\left\|\delta_{n}\right\|^{k} \\
& \leq C\|L f\|_{\infty}\left\|\delta_{n}\right\|^{k}, \quad n=1,2, \cdots,
\end{aligned}
$$

with $C$ an absolute constant. This proves the theorem for $\alpha=k$.

To establish the theorem for $0<a \leq k$, we use an intermediate space technique. If $f \in C[0,1]$ and $\epsilon>0$, there is a function $f_{\epsilon} \in C^{(k)}[0,1]$ for which

$$
\begin{gathered}
\left\|f-f_{\epsilon}\right\| \leq C_{1} \omega_{k}(f, \epsilon), \\
\left\|f_{\epsilon}^{(j)}\right\| \leq C_{2} \epsilon^{-k} \omega_{k}(f, \epsilon), \quad j=0, \cdots, k,
\end{gathered}
$$

with $C_{1}$ and $C_{2}$ constants depending only on $k$. For a proof of this fact, we refer the reader to the paper of G. Freud and V. Popov [2]. Freud and Popov have only stated (4.18) for the case $j=k$ but the estimate for other values of $j$ is immediate from their explicit construction of $f$.

Now, suppose $f \in C[0,1]$ and $0<a \leq k$ with

$$
\omega_{k}(f, t) \leq M t^{a} \text {. }
$$

For $n$ a positive integer take $\epsilon=\epsilon_{n}=\left\|\delta_{n}\right\|$ in (4.17) and (4.18). The function $f_{\epsilon_{n}}$ has $f_{\epsilon_{n}}^{(k-1)}$ absolutely continuous and $L f_{\epsilon_{n}} \in L_{\infty}$. In fact, if we express the operator $L^{n}$ as $L=\sum_{j=0}^{k} \alpha_{j}(x) D^{k}, D=d / d x$, with $\alpha_{j} \in C[0,1], j=0, \cdots, k$, then by using (4.19) it follows that

$$
\left\|L f_{\epsilon_{n}}\right\|_{\infty} \leq C_{3} \sup _{0 \leq j \leq k}\left\|f_{\epsilon_{n}}^{(j)}\right\| \leq C_{4} \epsilon_{n}^{-k} \omega_{k}\left(f, \epsilon_{n}\right) \leq C_{4} M \epsilon_{n}^{a-k}
$$

with $C_{3}$ and $C_{4}$ constants independent of $n$.

Let $S_{n}$ be a spline in $S\left(0, \delta_{n}\right)$ for which

$$
\left\|f_{\epsilon_{n}}-S_{n}\right\| \leq C C_{4} M \epsilon_{n}^{a}
$$

The existence of such a spline is guaranteed by the first part of our proof because 
of the estimate (4.20). Therefore, for $n=1,2, \cdots,\left\|f-s_{n}\right\| \leq\left\|f-f_{\epsilon_{n}}\right\|+\| f_{\epsilon_{n}}-$ $s_{n}\left\|\leq C_{1} M \epsilon_{n}^{a}+C C_{4} M \epsilon_{n}^{a} \leq C_{5}\right\| \delta_{n} \|^{a}$ where the term $\left\|f-f_{\epsilon_{n}}\right\|$ was estimated by (4.17). This completes the proof of the theorem.

5 The saturation class for algebraic polynomial splines with equally spaced knots. In this section, we suppose that the E.C.T. system is ordinary polynomials, i.e., $u_{i}(t)=t^{i}, i=0, \cdots, k-1$, and the knots are equally spaced $\left(\delta_{n}=\{i / n\}_{i=0}^{n}\right)$. In this case, it is possible to give a more precise characterization of the saturation class. We let $E_{n}(f)=E_{\delta_{n}}(f)$.

Theorem 4. For algebraic polynomial splines with equally spaced knots, the following two statements are equivalent for $f \in C[0,1]$ :

$$
\lim _{n \rightarrow \infty} n^{k} E_{n}(f)=2^{-2 k+1} \frac{M}{k !}
$$

$$
f^{(k-1)} \text { is absolutely continuous } f^{(k)} \in L_{\infty} \text { and }\left\|f^{(k)}\right\|_{\infty}=M .
$$

Proof. The proof is based on a well-known theorem of S. Bernstein (see G. Meinardus [7, p. 78]), which states that if $g \in C[-1,1]$, with $g^{(k-1)}$ absolutely continuous on $[-1,1]$ and $g^{(k)} \in L_{\infty}[-1,1]$ then the re exists a polynomial $P$ of degree $\leq k-1$ for which

$$
\|g-P\|[-1,1] \leq\left\|\widetilde{T}_{k}\right\|[-1,1] \cdot\left\|g^{(k)}\right\|_{\infty}[-1,1]=\left(2^{-k+1} / k !\right)\left\|g^{(k)}\right\|_{\infty}[-1,1]
$$

where $\widetilde{T}_{k}$ is the normalized Chebyshev polynomial

$$
\widetilde{T}_{k}(x)=\left(2^{-k+1} / k !\right) \cos (k \operatorname{arc} \cos x)=x^{k} / k !+\cdots .
$$

This result is usually only stated for $g^{(k)}$ continuous but is routinely extended to the general case $g^{(k)} \in L_{\infty}$ by approximating $g$ by $k$ times continuously differentiable functions $g_{\nu}$ with $\left\|g_{\nu}^{(k)}\right\|[-1,1] \leq\left\|g^{(k)}\right\|_{\infty}[-1,1]$.

We know from our inverse theorem (Theorem 1) that $E_{n}(f)=O\left(n^{-k}\right)$ if and only if $f^{(k-1)}$ is absolutely continuous and $f^{(k)} \in L_{\infty}$. For this reason, it will be enough to show that (5.2) implies (5.1). The other direction follows from Theorem 1 and the fact that (5.2) implies (5.1).

We first wish to show that (5.2) implies

$$
\varlimsup_{n \rightarrow \infty} n^{k} E_{n}(f) \leq \frac{2^{-2 k+1} M}{k !} .
$$

This is an easy consequence of (5.3). For each $0 \leq i \leq n-1$, there is a polynomial $P_{i, n}$ of degree at most $k-1$ for which 


$$
\left\|f-P_{i, n}\right\|[i / n,(i+1) / n] \leq 2^{-2 k+1} M / n^{k} k !
$$

Here, (5.6) is a restatement of $(5.3)$ for the interval $[i / n,(i+1) / n]$ as obtained via the usual transformation of $[i / n,(i+1) / n]$ to $[-1,1]$ and back again. Thus, the spline $S_{n} \in \mathcal{S}\left(\delta_{n}\right)$ defined to be $P_{i, n}$ on $[i / n,(i+1) / n), i=0, \cdots, n-1$, gives the estimate $E_{n}(f) \leq\left\|f-S_{n}\right\|[0,1] \leq 2^{-2 k+1} M / n^{k} k$ ! which, of course, shows (5.5).

We will now show that (5.2) implies that

$$
\lim _{n \rightarrow \infty} n^{k} E_{n}(f) \geq \frac{2^{-2 k+1} M}{k !}
$$

which will complete the proof of the theorem. Suppose (5.2) holds but

$$
\lim _{n \rightarrow \infty} n^{k} E_{n}(f)=\frac{2^{-2 k+1} M^{\prime}}{k !}
$$

with $M^{\prime}<M$. We will show that (5.8) implies the existence of a polynomial $Q$ of degree at most $k-1$ for which

$$
\left\|M x^{k} / k !-Q(x)\right\|[-1,1]<2^{-k+1} M / k ! \text {. }
$$

This will contradict the well-known minimality [7, p. 78] property of the Chebyshev polynomial $\widetilde{T}_{k}$ of having the smallest norm among all polynomials of degree $k$ with leading coefficient $1 / k$ !.

Let $\left(n_{j}\right)^{\prime}$ be a subsequence for which $\lim _{j \rightarrow \infty} n_{j}^{k} E_{n_{j}}(f)=2^{-2 k+1} M^{\prime} / k$ !. For $\epsilon=2^{-3 k+1}\left(M-M^{\prime}\right)$, choose $N$ so that for $n_{j} \geq N$

$$
n_{j}^{k} E_{n_{j}}(f)<2^{-2 k+1}\left(M^{\prime}+\epsilon\right) / k ! \text {. }
$$

Since $\left\|f^{(k)}\right\|_{\infty}=M$, there is a point $x_{0}$ for which $f^{(k)}\left(x_{0}\right) \geq M-\epsilon / 2$. Here, we may have to work with $-f$ in place of $f$ in order to have the inequality read in the direction we want. There is a $\delta>0$ such that

$$
(M-\epsilon)\left(x-x_{0}\right) \leq f^{(k-1)}(x)-f^{(k-1)}\left(x_{0}\right) \leq M\left(x-x_{0}\right), \text { for } x_{0} \leq x \leq x_{0}+\delta .
$$

If we integrate the inequality (5.11) $k-1$ times, we find

$$
\begin{aligned}
(M-\epsilon)\left(x-x_{0}\right)^{k} / k ! \leq & f(x)-f\left(x_{0}\right)-f^{\prime}\left(x_{0}\right)\left(x-x_{0}\right) \\
& -\cdots-f^{(k-1)}\left(x_{0}\right)\left(x-x_{0}\right)^{k-1 / k !} \\
\leq & M\left(x-x_{0}\right)^{k} / k !, \quad \text { for } x_{0} \leq x \leq x_{0}+\delta .
\end{aligned}
$$

This last inequality shows that there is a polynomial $P_{1}$ of degree $\leq k-1$ such that for each $0<\eta \leq \delta$ 


$$
\left\|M x^{k} / k !-f(x)-P_{1}(x)\right\|\left[x_{0}, x_{0}+\eta\right] \leq \epsilon \eta^{k} / k ! \text {. }
$$

Now, choose $n_{j} \geq \min \left(N, 2 \delta^{-1}\right)$. Then, for some $0<i \leq n_{j}-1$, we have $\left[i / n_{j},(i+1) / n_{j}\right] \subseteq\left[x_{0}, x_{0}+2 / n_{j}\right] \subseteq\left[x_{0}, x_{0}+\delta\right]$. Taking $\eta=2 n_{j}^{-1}$ in (5.12) gives

$$
\left\|M x^{k} / k !-f(x)-P_{1}(x)\right\|\left[i / n_{j},(i+1) / n_{j}\right] \leq \epsilon 2^{k} / n_{j}^{k} k ! .
$$

Since $E_{n_{j}}(f)<2^{-2 k+1}\left(M^{\prime}+\epsilon\right) / n_{j}^{k} k$ !, there is a polynomial $P_{2}$ of degree at most $k-1$ such

$$
\left\|f(x)-P_{2}(x)\right\|\left[i / n_{j},(i+1) / n_{j}\right] \leq 2^{-2 k+1}\left(M^{\prime}+\epsilon\right) / n_{j}^{k} k ! .
$$

Using (5.14) in (5.13) gives

(5.15) $\left\|\frac{M x^{k}}{k !}-P_{1}(x)-P_{2}(x)\right\|\left[\frac{i}{n_{j}}, \frac{i+1}{n_{j}}\right] \leq \frac{2^{-2 k+1} M^{\prime}}{n_{j}^{k} k !}+\frac{2^{k+1} \epsilon}{n_{j}^{k} k !}<\frac{2^{-2 k+1} M}{n_{j}^{k} k !}$

where in the last inequality we have used our choice of $\epsilon$. Finally, $P_{1}-P_{2}$ is a polynomial of degree $\leq k-1$ and so transforming (5.15) to the interval $[-1,1]$ establishes the existence of the polynomial $Q$ in (5.9). The proof is complete.

\section{REFERENCES} (to appear).

1. G. Butler and F. Richards, An $L_{p}$-saturation theorem for splines, Canad. J. Math.

2. G. Freud and V. Popov, On approximation by spline functions, Proc. Conference on Constructive Theory of Functions, Budapest, 1968, pp. 163-172.

3. D. Gaier, Saturation bei Spline-Approximation und Quadratur, Numer. Math. 16 (1970), 129-140. MR $42 \# 8692$.

4. S. Karlin, Total positivity. Vol. I, Stanford Univ. Press, Stanford, Calif., 1968. MR 37 \#5667.

5. S. Karlin and W. J. Studden, Tchebycheff systems: With applications in analysis and statistics, Pure and Appl. Math., vol. 15, Interscience, New York, 1966. MR 34 \#4757.

6. G. G. Lorentz, Approximation of functions, Holt, Rinehart and Win ston, New York, 1966. MR $35 \# 4642$.

7. G. Meinardus, Approximation von Funktionen ud ihre numerische Behandlung, Springer Tracts in Natural Philosophy, vol. 4, Springer-Verlag, Berlin and New York, 1964. MR $31 \# 547$.

8. V. A. Popov and Bl. K. Sendov, The classes that are characterized by the best approximation by spline functions, Mat. Zametki 8 (1970), 137-148= Math. Notes 8 (1970), 550-557. MR 43 \#5224.

9. F. Richards, On the saturation class for spline functions, Proc. Amer. Math. Soc. 33 (1972), 471-476. 
10. K. Scherer, On the best approximation of continuous functions by splines, SIAM J. Numer. Anal. 7 (1970), 418-423. MR $42 \# 2634$.

11. A. F. Timan, The ory of approximation of functions of a real variable, Fizmatgiz, Moscow, 1960; English transl., Internat. Series of Monographs in Pure and Appl. Math., vol. 34, Macmillan, New York, 1963. MR 22 \#8257; MR 33 \#465. 48063

DEPARTMENT OF MATHEMATICS, OAKLAND UNIVERSITY, ROCHESTER, MICHIGAN

DEPARTMENT OF MATHEMATICS, UNIVERSITY OF ALBERTA, EDMONTON, ALBERTA, CANADA 